\title{
Synthesis of (4-chlorobenzylidene)-(2-chloropyridi-3-yl)amine
}

\section{A. A. Jarrahpour* and M. Zarei}

Department of Chemistry, College of Sciences, Shiraz University, Shiraz 71454, Iran Tel.0098 711 2284822, Fax: 0098711 2280926, e-mail: aliasghar6683@yahoo.com, jarrah@,susc.ac.ir

Received: 2 June 2005 / Accepted: 22 September 2005 / Published: 1 October 2005

Keywords: Schiff bases , 3-amino-2-chloropyridine , $p$-chlorobenzaldehyde , biological activity .

Schiff bases are widely in use for synthetic purposes both by organic and inorganic chemists. ${ }^{1}$ They are used as biological, analytical, polymer and liquid crystalline materials. ${ }^{2}$ Schiff bases are reported to show a variety of biological activities such as antibacterial ${ }^{3-5}$, antifungal ${ }^{6-7}$, anticancer ${ }^{8-9}$ and herbicidal ${ }^{10}$ activities . Pyridinium compounds have biological activities ${ }^{11}$ such as antifungal ${ }^{12}$ and antibacterial ${ }^{12-13}$ activities. The presence of a chloro moiety in different types of compounds causes them to exhibit pesticidal activity. ${ }^{10}$<smiles>Nc1cccnc1Cl</smiles>

1

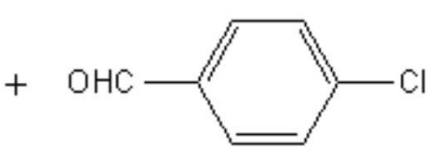

2<smiles>Clc1ccc(/C=N/c2cccnc2Cl)cc1</smiles>

3

A mixture of 3-amino-2-chloro pyridine $1(1.28 \mathrm{~g}, 10 \mathrm{mmol})$, 4-chlorobenzaldehyde $\mathbf{2}(1.40 \mathrm{~g}, 10 \mathrm{mmol})$ and anhydrous sodium sulfate $(4.00 \mathrm{~g})$ in dry dichloromethane $(40.00 \mathrm{~mL})$ was stirred at room temperature for seven hours. The suspension was filtered and washed with dichloromethane. The solvent was evaporated and new schiff base 3 was formed, which was washed with $10 \mathrm{~mL}$ acetic acid 5\%, $10 \mathrm{~mL} \mathrm{NaHCO}_{3} 5 \%$ and water, successively, $(2.04 \mathrm{~g}, 82 \%)$.

Melting point: $54-56^{\circ} \mathrm{C}$.

IR $\left(\mathrm{KBr}, \mathrm{cm}^{-1}\right): 1600.1(\mathrm{CH}=\mathrm{N}) ; 1624.0(\mathrm{C}=\mathrm{N}$ pyridine ring $)$.

${ }^{1} \mathrm{H}-\mathrm{NMR}\left(250 \mathrm{MHz}, \mathrm{CDCl}_{3}\right): \delta=6.97-8.17(7 \mathrm{H}, \mathrm{m}, 2 \mathrm{Ph}) ; 8.31(1 \mathrm{H}, \mathrm{s}, \mathrm{HC}=\mathrm{N})$.

${ }^{13} \mathrm{C}-\mathrm{NMR}$ (62.9 MHz, $\left.\mathrm{CDCl}_{3}\right): \delta=123.55-146.49$ (aromatic carbons); $162.55(\mathrm{HC}=\mathrm{N})$.

MS (m/z, \%): $251\left(\left[\mathrm{M}^{+}\right], 63.3\right) ; 250(\mathrm{M}-1,100.0) ; 215\left(\mathrm{C}_{5} \mathrm{H}_{3} \mathrm{NClN}=\mathrm{CC}_{6} \mathrm{H}_{4}, 14.8\right) ; 139\left(\mathrm{C}_{5} \mathrm{H}_{3} \mathrm{NClN}=\mathrm{C}, 15.4\right) ; 112$ $\left(\mathrm{C}_{5} \mathrm{H}_{3} \mathrm{NCl}, 52.2\right), 89\left(\mathrm{C}_{5} \mathrm{H}_{3} \mathrm{NN}, 19.4\right) ; 77\left(\mathrm{C}_{5} \mathrm{H}_{3} \mathrm{~N}, 15.1\right)$.

\section{Acknowledgment}

The authors thank the Shiraz University Research Council for financial support (Grant No. 83-GR-SC-31 and 84-GR-SC-23 ).

\section{References}

1. K. Arora, A. Gupta, D. D. Agarwall Asian. J. Chem. 2002, 14, 1611.

2. K. Tanaka, R. Shiraishi Green Chem. 2000, 2, 272.

3. A. Shaikh kabeer, M. A. Baseer, N. A. Mote Asian J. Chem. 2001, 13(2), 496.

4. S. N. Pandeya, D. Sriram, G. Nath, E. De Clercq Il Farmaco 1999 ,54, 624.

5. Afaf. H. El-masry, H. H. Fahmy, S. H. Ali Abdelwahed Molecules 2000, 5, 1429.

6. P. G. More, R. B. Bhalvankar, S. C. Pattar J. Indian Chem. Soc. 2001, 78, 474.

7. W. M. Singh, B. C. Dash Pesticides 1988, 22 (11), 33. 
8. S. B. Desai, P. B. Desai, K. R. Desai Hetrocycl. Commun. 2001, 7 (1), 83.

9. P. Phatak, V. S. Jolly, K. P.Sharma Orient. J. Chem. 2000, 16 (3), 493.

10. S. Samadhiya, A. Halve Orient. J. Chem. 2001, 17 (1), 119.

11. Kristina Sepcic J. Toxicol., Toxin Rev. 2000, 19 (2), 139.

12. W. Sliwa, B. Mianowska Hetrocycles 1989, 29 (3), 557.

13. I. Durta, R. Dinica, E. Bacu, M. Anderi Chem. Abstr. 2000, 132, 205326 b.

Sample Availability: Available from MDPI.

(C) 2005 MDPI. All rights reserved. 